\title{
TP53 and CDKN2A mutations in patients with early-stage lung squamous cell carcinoma: an analysis of the correlations and prognostic outcomes
}

\author{
Peiyuan Wang ${ }^{1,2,3 \#}$, Feng Wang ${ }^{1 \#}$, Hao He ${ }^{1}$, Yujie Chen ${ }^{1}$, Hui Lin ${ }^{1}$, Peng Chen ${ }^{1}$, Xiaofeng Chen ${ }^{1}$, \\ Shuoyan Liu ${ }^{1}$
}

${ }^{1}$ Department of Thoracic Oncology Surgery, Fujian Medical University Cancer Hospital, Fujian Cancer Hospital, Fuzhou, China; ${ }^{2}$ Fujian Key Laboratory of Translational Cancer Medicine, Fuzhou, China; ${ }^{3}$ Fujian Provincial Key Laboratory of Tumor Biotherapy, Fuzhou, China

Contributions: (I) Conception and design: P Wang; (II) Administrative support: S Liu, F Wang; (III) Provision of study materials or patients: H He, Y Chen; (IV) Collection and assembly of data: H Lin, P Chen, X Chen; (V) Data analysis and interpretation: P Wang, F Wang; (VI) Manuscript writing: All authors; (VII) Final approval of manuscript: All authors.

\#These authors contributed equally to this work.

Correspondence to: Shuoyan Liu. Department of Thoracic Oncology Surgery, Fujian Medical University Cancer Hospital, Fujian Cancer Hospital, Fuzhou, China. Email: shuoyanliu2010@163.com.

Background: Lung squamous cell carcinoma (LUSC) is characterized by frequent mutations of tumor protein p53 (TP53) and cyclin dependent kinase inhibitor 2A (CDKN2A). However, to date, the impact of TP53/CDKN2A status on the clinical outcome of patients with early-stage LUSC is unclear.

Methods: Tissue samples from 16 early-stage, surgically resected LUSCs were analyzed by next-generation sequencing (NGS). Information regarding TP53 and CDKN2A alterations and patient survival time was downloaded from The Cancer Genome Atlas (TCGA) database. The associations between TP53 and CDKN2A status and tumor characteristics, outcomes including overall survival (OS) and disease-free survival (DFS), and mutation counts were investigated.

Results: TP53 and CDKN2A exhibited a high frequency of somatic mutations in early-stage LUSC in our center. Data for 1,176 samples were collected from TCGA. CDKN2A mutation status was associated with TP53 mutation status $(\mathrm{P}=0.040)$. TP53 mutation was a favorable prognostic factor for early-stage LUSC. The OS times of patients with wild-type and mutated TP53 were 28.94 and 60.48 months, respectively $(\mathrm{P}=0.002)$. In contrast, CDKN2A mutations were significantly associated with a shorter survival time in earlystage LUSC. The OS times for wild-type and mutated CDKN2A patients were 62.81 and 37.55 months, respectively $(\mathrm{P}=0.026)$. Patients with TP53 mutations had higher total mutation counts compared to patients with wild-type TP53. Furthermore, OS was significantly shorter in patients with a low mutation count compared to patients with a median or high mutation count.

Conclusions: Early-stage LUSC patients with TP53 mutations had a longer OS, while those with CDKN2A mutations had a shorter OS. Furthermore, patients with TP53 mutation/CDKN2A wild-type status had a longer OS. CDKN2A mutation is a vital indicator for prognostic assessment according to TP53 status. The prolonged survival of patients with TP53 mutations may be due to their high mutation counts. Larger datasets are required to validate these observations.

Keywords: Tumor protein p53 (TP53); cyclin dependent kinase inhibitor 2A (CDKN2A); lung squamous cell carcinoma (LUSC); mutation; prognosis

Submitted Jun 22, 2021. Accepted for publication Aug 16, 2021.

doi: 10.21037/atm-21-3709

View this article at: https://dx.doi.org/10.21037/atm-21-3709

\footnotetext{
^ ORCID: 0000-0001-5544-7457.
} 


\section{Introduction}

Lung squamous cell carcinoma (LUSC) accounts for 20 $30 \%$ of non-small-cell lung cancers (NSCLCs) and results in approximately 400,000 deaths annually in the United States. Unfortunately, to date, very few personalized therapies have been developed for LUSC due to the limited understanding of the molecular targets (1). Previous profiling efforts have demonstrated that mutations in tumor protein p53 (TP53) represent the most frequent $(81 \%)$ genomic alteration found in LUSC (2). TP53 encodes the tumor suppressor protein $\mathrm{p} 53$, binds directly to chromatin in the nucleus, and plays an important role in the regulation of the cell cycle, apoptosis, autophagy, and DNA repair in response to oncogenic stress (3). The position, nature, and functional effects of mutations on protein structure and activity have led to a recent classification of TP53 mutations, and it is now recognized that various classes of mutations have differential prognostic effects. However, data on the prognostic or predictive effects of TP53 status in NSCLC are limited and inconclusive (4). To date, there is still a paucity of drugs approved for targeting TP53 mutations in cancer patients, and the prognostic value of TP53 in early-stage LUSC is unclear. Thus, this study examined the prognostic value of TP53 in early-stage LUSC.

Cyclin dependent kinase inhibitor 2A $(C D K N 2 A)$, a known tumor suppressor gene that encodes the $\mathrm{p} 16^{\mathrm{INK} 4 \mathrm{~A}}$ and $\mathrm{p} 14^{\mathrm{ARF}}$ proteins, is inactivated in $72 \%$ of LUSC cases (2). Patients who are carriers of certain CDKN2A mutations show increased risks of malignant neoplasms, particularly pancreatic, lung, and head and neck cancers (5). LUSC is characterized by frequent TP 53 mutations and CDKN2A alterations $(2,6)$. Previous studies have reported that the degradation of the $\mathrm{p} 53$ protein by the ubiquitin pathway is mediated by its binding to mouse double minute 2 (MDM2). However, the expression of MDM2 mRNA and protein is negatively regulated by $\mathrm{p} 14^{\mathrm{ARF}}$ in the nucleus $(7,8)$. While these alterations have increased our understanding of the molecular pathology of LUSC, the impact of TP53/CDKN2A status on the clinical outcomes of patients with early-stage LUSC is unclear.

This study analyzed the mutational landscape of 16 earlystage, surgically resected LUSC patients using targeted next-generation sequencing (NGS) encompassing 59-1,021 cancer-related genes. Furthermore, we utilized a well annotated specimen set that permits analysis of mutations, alone or in combination, with outcome. This study aimed to evaluate the association of TP53 and CDKN2A status, as well as the prognostic value of these two genes combined in early-stage, surgically resected LUSC. We present the following article in accordance with the REMARK reporting checklist (available at https://dx.doi.org/10.21037/ atm-21-3709).

\section{Methods}

\section{Patients and samples}

Sixteen early-stage, surgically resected LUSC samples were obtained from the Fujian Cancer Hospital in Fuzhou, China, from August 2018 to August 2019. All patients provided written informed consent and received NGS testing at the Geneplus-Beijing Institute. NGS testing covered approximately 1.4 Mbp genomic regions of 1,021 cancer-related genes (or approximately $230 \mathrm{Kbp}$ genomic regions of 59 genes for some patients) (Table S1). All procedures performed in this study involving human participants were in accordance with the Declaration of Helsinki (as revised in 2013). The study was approved by regional ethics board of Fujian Cancer Hospital (No.: SQ2020-055-01) and informed consent was taken from all the patients.

\section{Gene expression databases}

Information regarding TP53 and CDKN2A alterations and survival times in patients with LUSC was downloaded from The Cancer Genome Atlas (TCGA), an open access database that is publicly available at http://www.cbioportal. org. The Lung Squamous Cell Carcinoma (TCGA, Firehose Legacy), Lung Squamous Cell Carcinoma (TCGA, Nature 2012), and Lung Squamous Cell Carcinoma (TCGA, PanCancer Atlas) datasets were selected as the data source as they contained only early-stage, surgically resected LUSC samples. There was a total of 1176 LUSC samples (available online: https://cdn.amegroups.cn/static/ public/atm-21-3709-1.xlsx). The gene set of interest, "TP53 CDKN2A", was entered in the input box. Mutation and survival data were downloaded from the cBioPortal website after submitting the query regarding "TP53 CDKN2A" in the input box. Data were merged according to the unique patient ID, such as "TCGA-18-3406-01". Altogether, 841 pieces of mutation data and 979 pieces of survival data were downloaded. Analysis of the data revealed 349 pieces of duplicated patient data, which were discarded. The duplication was largely due to overlapping data with another 

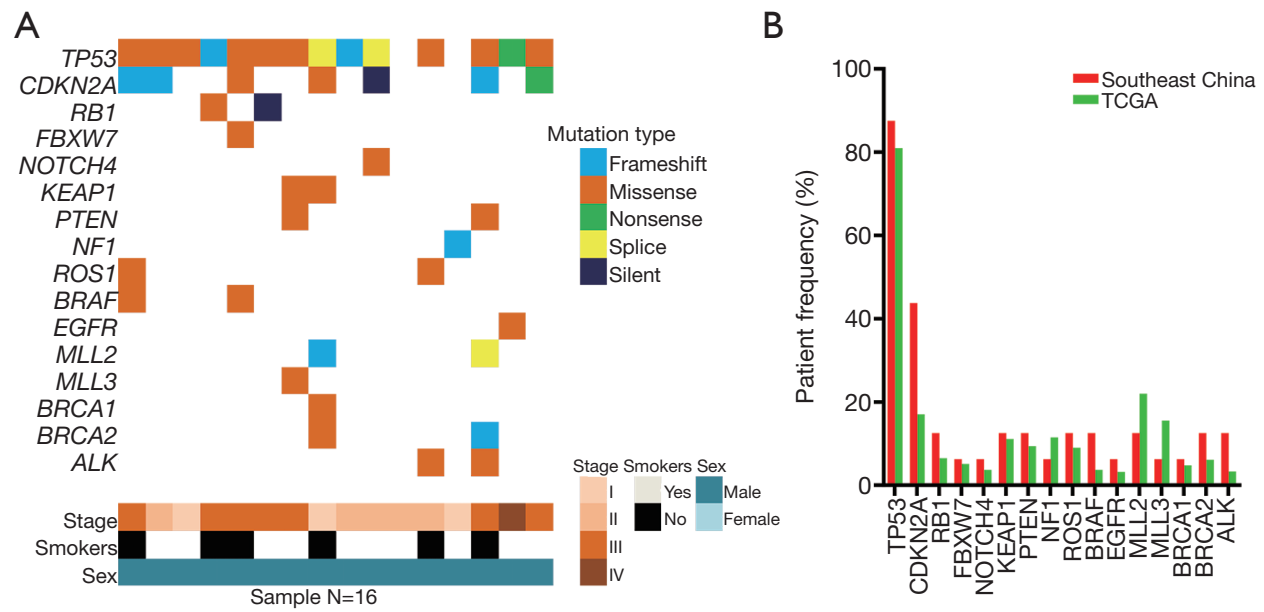

Figure 1 Significantly mutated genes observed in early-stage, surgically resected lung squamous cell carcinoma (LUSC) samples obtained in our center. (A) The top 16 significantly mutated genes in LUSC samples. (B) A comparison of the mutation frequencies of significantly mutated genes between the southeastern China cohort and The Cancer Genome Atlas (TCGA) cohort of LUSC patients.

selected study. After the merge, there were 492 pieces of data from early-stage LUSC patients. Each piece of data contained the mutation type of CDKN2A and TP53 as well as the survival time of the patient. The TP53 and CDKN2A mutations were divided into different groups based on the different exons containing the mutations. However, due to limited information from the cBioPortal database, there were 13 cases without mutation counts in TP53-wild-type patients and 10 cases without mutation counts in TP53mutated patients. No statements of approval or informed consent were required for this section of the study, as all data was obtained from an open access database.

\section{Statistical methods}

Fisher's exact test and the Mann-Whitney test were utilized to analyze the categorical and continuous variables. Survival curves were analyzed using the Kaplan-Meier method and log-rank tests. The Cox proportional hazards model was used to evaluate associations between clinicopathological characteristics and patient survival. Overall survival (OS) and disease-free survival (DFS) data were obtained from the cBioPortal website directly. Statistical analyses were performed using GraphPad Prism 5.0. The statistical significance (alpha-value) threshold was fixed at 0.05 , and all $P$ values were three-sided.

\section{Results}

\section{TP53 and CDKN2A exhibited a bigher frequency of somatic mutations than other cancer-related genes}

A retrospective study was conducted on 16 LUSC patients involving genomic profiling via targeted NGS encompassing 59-1,021 cancer-related genes. Among LUSC patients, the rate of TP53 mutation was $87.5 \%(14 / 16)$, while the rate of CDKN2A mutation was $43.8 \%$ (7/16). Interestingly, CDKN2A mutations were accompanied by TP53 mutations. TP53 and CDKN2A were among the most frequently mutated genes, whereas F-box and WD repeat domain containing 7 (FBXW7), notch receptor 4 (NOTCH4), epidermal growth factor receptor (EGFR), BRCA1 DNA repair associated (BRCA1) had lower mutation rates in our study cohort (Figure 1A). The mutation frequencies of TP53, NOTCH4, kelch like ECH associated protein 1 (KEAP1), phosphatase and tensin homolog (PTEN), ROS proto-oncogene 1, receptor tyrosine kinase (ROS1), EGFR, and BRCA1 were comparable with those in the TCGA 

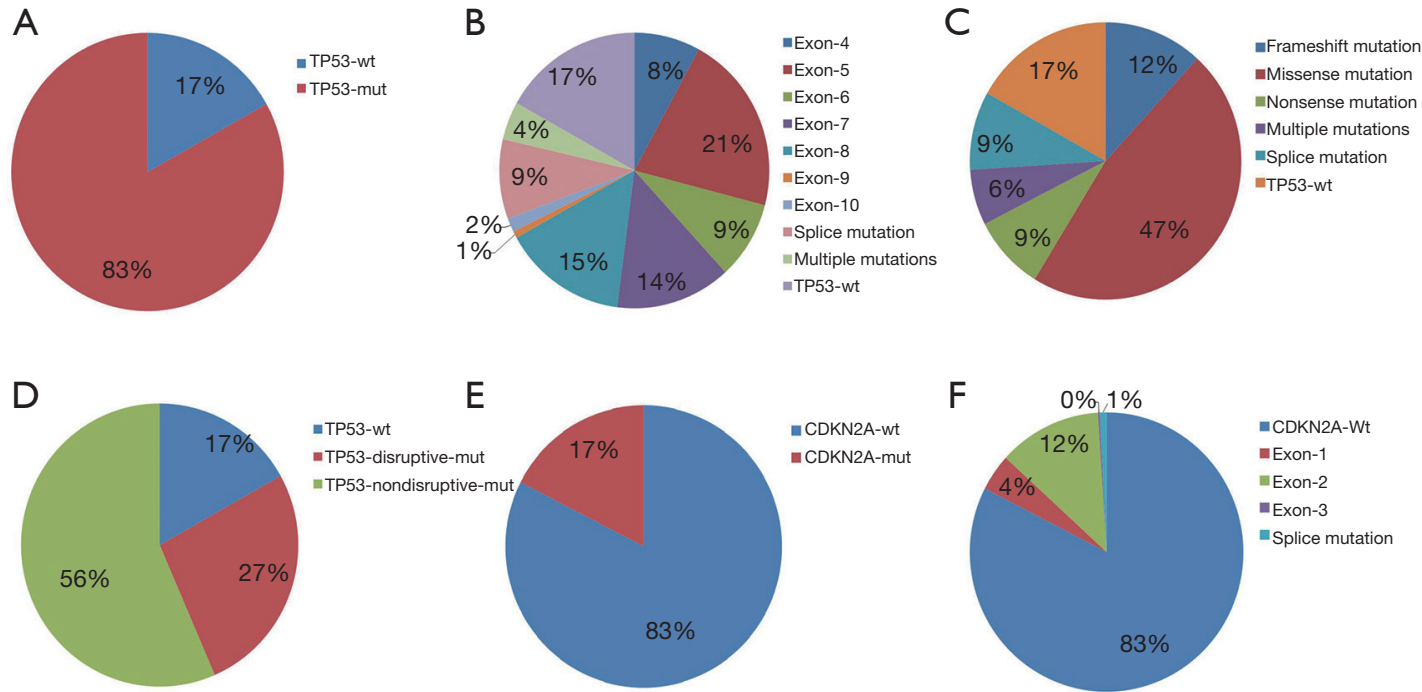

Figure 2 The distribution of tumor protein p53 (TP53) and cyclin dependent kinase inhibitor 2A (CDKN2A) mutations in early-stage lung squamous cell carcinoma (LUSC). (A) The distribution of TP53 wild type (wt) or mutated type (mut). (B) The distribution of different TP53 mutation sites. (C) The distribution of different TP53 mutation types. (D) The distribution of TP53 mutation status divided into wild type, disruptive mutation, and nondisruptive mutation groups. (E) The distribution of CDKN2A wild type (wt) or mutated type (mut). (F) The distribution of different CDKN2A mutation sites.

data (Figure 1B). Patients with early-stage LUSC exhibited higher TP53 and CDKN2A mutation frequencies compared to other cancer-related genes.

\section{TP53 mutation and CDKN2A mutation profiling and patient characteristics}

In the 492 early-stage LUSC patients, the mutation rate of TP53 was $83.13 \%$ (409/492). Exons $4-8$ were the most frequent mutation sites for TP53, accounting for $66.9 \%$ of all mutations (329/492). Exons 9 and 10 were rarely mutated, accounting for $2.4 \%$ of all mutations (12/492). Multiple mutations occurred in $4.5 \%$ of patients $(22 / 492)$, and $9.3 \%(46 / 492)$ of mutations could not be classified (Figure 2A,2B). TP53 mutations, mainly missense mutations, were the most common mutations in early-stage LUSC (Figure 2C).

In another classification, TP53 mutation status was divided into wild type, disruptive mutations, and nondisruptive mutations, as previously described (9). A total of 131 patients $(27 \%)$ had TP53 disruptive mutations, and 278 patients $(56 \%)$ showed nondisruptive TP53 mutations (Figure 2D). Patient and tumor characteristics based on TP53 status are shown in Table S2. Dual TP53/CDKN2A mutations were observed in 78 patients (15.8\%). CDKN2A mutation status was associated with TP53 mutation status $(\mathrm{P}=0.040)$.

Of the 492 patients, 406 patients did not have CDKN2A mutations, 21 patients had exon 1 mutations, 60 had exon 2 mutations, 1 had exon 3 mutations, and 4 had splice mutations (Figure $2 E, 2 F$ ). Patient and tumor characteristics based on CDKN2A status are shown in Table S3. There were no statistically significant differences in tumor characteristics.

\section{Kaplan-Meier analyses of survival time according to TP53 status in early-stage LUSC}

Consistent with the prognostic capacity of tumor staging, among the entire cohort of 492 patients, the KaplanMeier survival curve indicated that patients with different tumor stages had significantly different $\mathrm{OS}$ times $(\mathrm{P}=0.009$; Figure $3 A$ ). Similarly, there was a significant difference in OS based on TP53 mutant or wild-type status with distinct tumor staging $(\mathrm{P}=0.020$ and $\mathrm{P}=0.025$, respectively) (Figure 3B,3C). However, the year of initial diagnosis did not significantly affect prognosis $(\mathrm{P}=0.595$; Figure $\mathrm{S} 1 \mathrm{~A})$.

Furthermore, TP53 mutation was a positive prognostic factor for OS and DFS in early-stage LUSC (Figure 3D and Figure S1B). The estimated OS times for patients with 

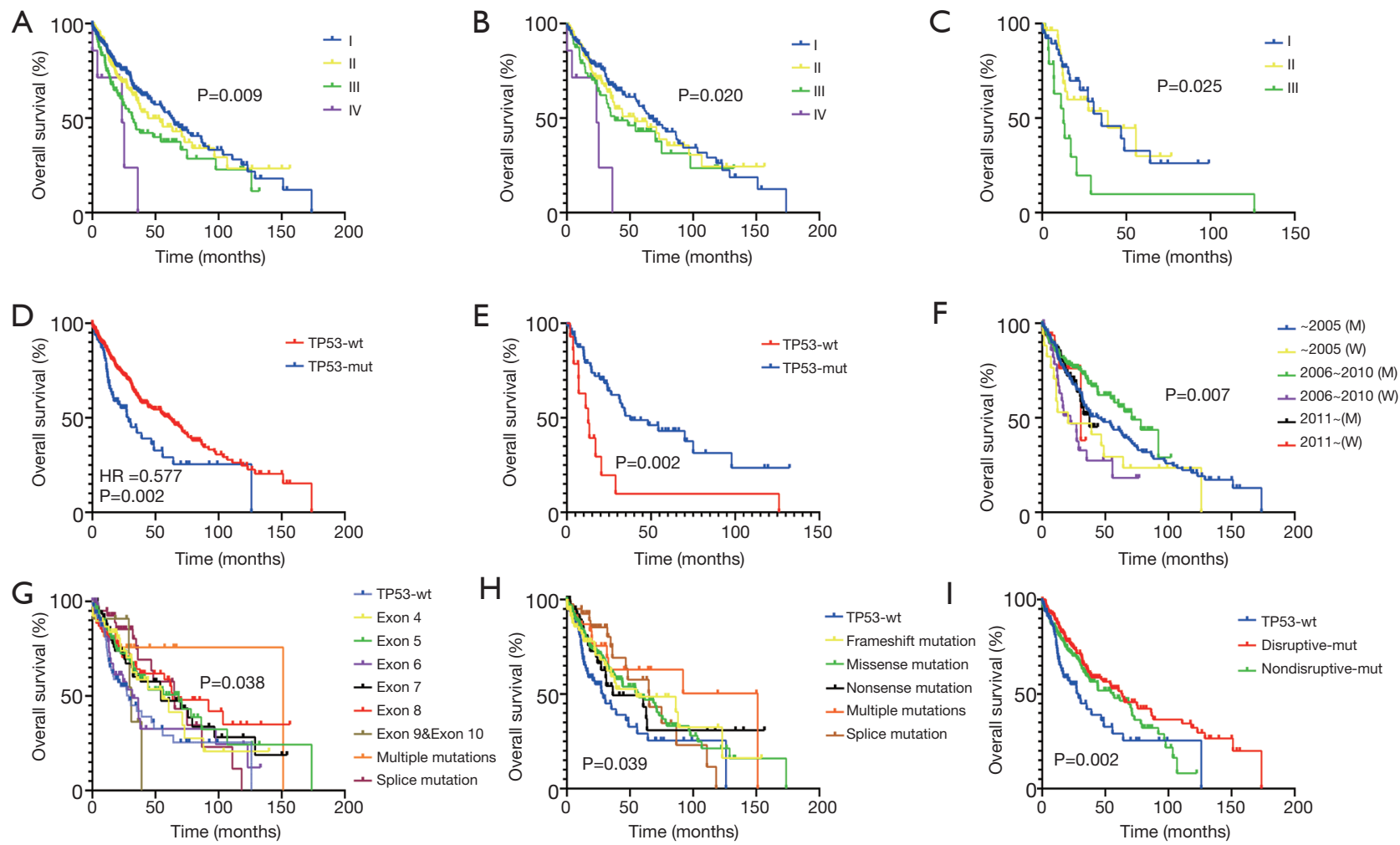

Figure 3 Survival curves of patients carrying tumor protein p53 (TP53) mutations. (A) Overall survival (OS) in different tumor stages. (B) OS in TP53-mutated tumors of different stages. (C) OS in TP53 wild-type tumors of different stages. (D) OS in TP53 wild-type and TP53mutated patients. (E) OS in stage III patients carrying wild-type or mutated TP53. (F) OS in patients carrying wild-type or mutated TP53 depending on the year of initial diagnosis. (G) OS in patients with wild-type and mutated TP53 subdivided according to mutation site. (H) OS in patients with wild-type and mutated TP53 subdivided according to mutation type. (I) OS in patients with wild-type and mutated TP53 subdivided into disruptive or nondisruptive mutation types.

wild-type TP53 and mutated TP53 were 28.94 months and 60.48 months, respectively [hazard ratio (HR) 0.577; 95\% confidence interval (CI), 0.390 to $0.878 ; \mathrm{P}=0.002$ ]. This prompted us to investigate the association between patient survival time and tumor stage as well as TP53 mutations. In stage III patients, OS was affected by TP53 status (HR 4.21; 95\% CI, 1.68 to 10.56 ; $\mathrm{P}=0.002$ for OS), but no significant difference was identified in stage I-II patients (Figure $3 E$ and Figure S1C,S1D). In addition, OS for the entire cohort was influenced by the year of initial diagnosis according to TP53 status $(\mathrm{P}=0.007$; Figure $3 F)$.

TP5 3 mutations were divided according to the affected exons. Patients with different mutated exons had significantly different $\mathrm{OS}$ times $(\mathrm{P}=0.038$; Figure $3 G)$. However, the difference in DFS was not statistically significant (Figure S1E). Diverse types of TP53 mutations can occur, and the TP53 mutation type can affect the prognosis of LUSC patients $(\mathrm{P}=0.045$ for OS and $\mathrm{P}=0.039$ for PFS; Figure $3 H$ and Figure $\mathrm{S} 1 \mathrm{~F})$. In another classification, TP53 mutation status was divided into wild type, disruptive mutations, and nondisruptive mutations. The difference in survival between these mutation groups was also statistically significant $(\mathrm{P}=0.002$ for $\mathrm{OS}$ and $\mathrm{P}=0.039$ for PFS; Figure 3I).

\section{Survival analysis of TP53 and CDKN2A status in early- stage LUSC}

Few studies have demonstrated the prognostic impact of CDKN2A mutations in early-stage LUSC. The results demonstrated that the CDKN2A mutation was a negative prognostic factor in early-stage LUSC (Figure 4A). The estimated OS times for patients with wild-type CDKN2A and mutated CDKN2A were 62.81 months and 37.55 months, 

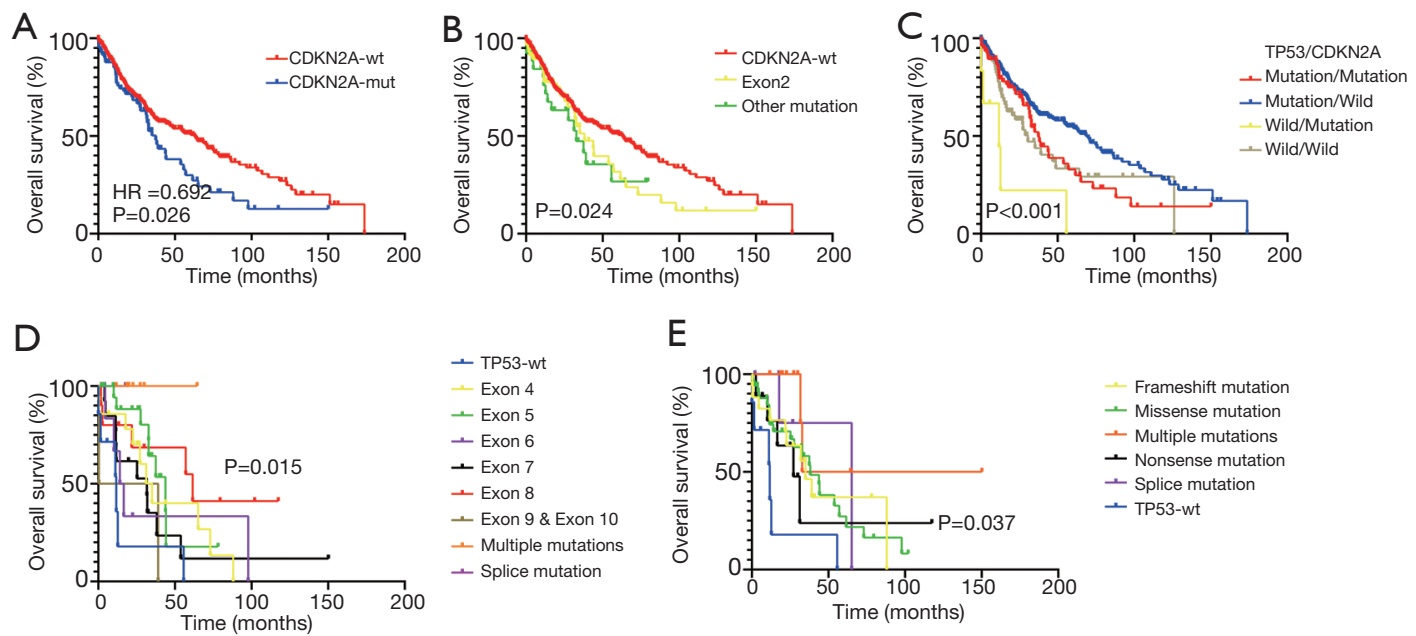

Figure 4 Survival curves of patients carrying cyclin dependent kinase inhibitor 2A (CDKN2A) and tumor protein p53 (TP53) mutations. (A) Overall survival (OS) in patients with wild-type and mutated CDKN2A. (B) OS in patients with wild-type and mutated CDKN2A subdivided according to mutation site. (C) OS of patients in different CDKN2A/TP53 mutation groups. (D) OS according to different TP53 mutation sites in CDKN2A-mutated patients. (E) OS according to different TP53 mutation types in CDKN2A-mutated patients.

respectively (HR $0.692 ; 95 \% \mathrm{CI}, 0.479$ to $0.998 ; \mathrm{P}=0.026$ ). DFS was not influenced by CDKN2A status (HR 0.823; $95 \%$ CI, 0.596 to $1.138 ; \mathrm{P}=0.209$; Figure $\mathrm{S} 2 \mathrm{~A}$ ). The mutation type was further divided according to the affected exons, and the difference between exon groups was statistically significant for $\mathrm{OS}(\mathrm{P}=0.024$; Figure $4 B)$. Again, $\mathrm{DFS}$ was not significantly different between the exon groups $(\mathrm{P}=0.197$; Figure S2B). The patients were further divided into different groups based on the mutation type of CDKN2A (wild type or mutated) and TP53 (wild type or mutated). The results revealed that the survival of the 4 different groups was significantly different $(\mathrm{P}<0.001$ for OS and DFS; Figure $4 C$ and Figure S2C). Patients with TP53 mutated/CDKN2A wild-type status showed longer OS and DFS compared to patients in the other 3 groups. This suggested that TP53 mutation and CDKN2A mutation types are prognostic factors in early-stage LUSC.

The prognostic value of CDKN2A and TP53 mutation types was further investigated. Survival curves of CDKN2Amutated patients indicated that TP53 wild-type patients had a poor prognosis $(\mathrm{P}=0.015$ for different mutation sites; $\mathrm{P}=0.037$ for different mutation types; Figure $4 D, 4 E$ ). However, in CDKN2A wild-type patients, OS was not influenced by TP53 status ( $\mathrm{P}=0.219$ for different mutation sites in different exons; $\mathrm{P}=0.154$ for different mutation types; Figure S2D,S2E). Interestingly, when TP53 mutation status was divided into wild type, disruptive mutations, and nondisruptive mutations, OS was not influenced by CDKN2A status (Figure S2F,S2G).

\section{Correlation of mutation counts and survival time}

Oncogenic stress triggers the DNA damage response which involves $\mathrm{p} 53$-mediated DNA repair to trigger cell cycle arrest and cell death by apoptosis or senescence (10). When TP53 is mutated, more mutations may occur. The association between mutation counts during early-stage LUSC and at different tumor stages was investigated. Different stages exhibited similar mutation frequencies. Total mutation counts were not influenced by stage regardless of TP53 status (Figure S3A-S3C). Interestingly, patients with mutated TP53 harbored more total mutations (Figure 5A). However, the CDKN2A wild-type group and CDKN2A-mutated group exhibited similar mutation counts (Figure S3D). In addition to the exon 4 mutation, different TP53 mutation sites were related to higher mutation counts compared to wild-type TP53 (Figure 5B). Similarly, patients with TP53 frameshift mutation, missense mutation, and multiple mutations had more mutations, with the exception of splice mutations and nonsense mutations (Figure 5C). In addition, patients with disruptive and nondisruptive TP53 mutations all presented with higher mutation counts than TP53 wild-type patients (Figure 5D). Moreover, compared to the TP53 and CDKN2A wild-type cohorts, the TP53 

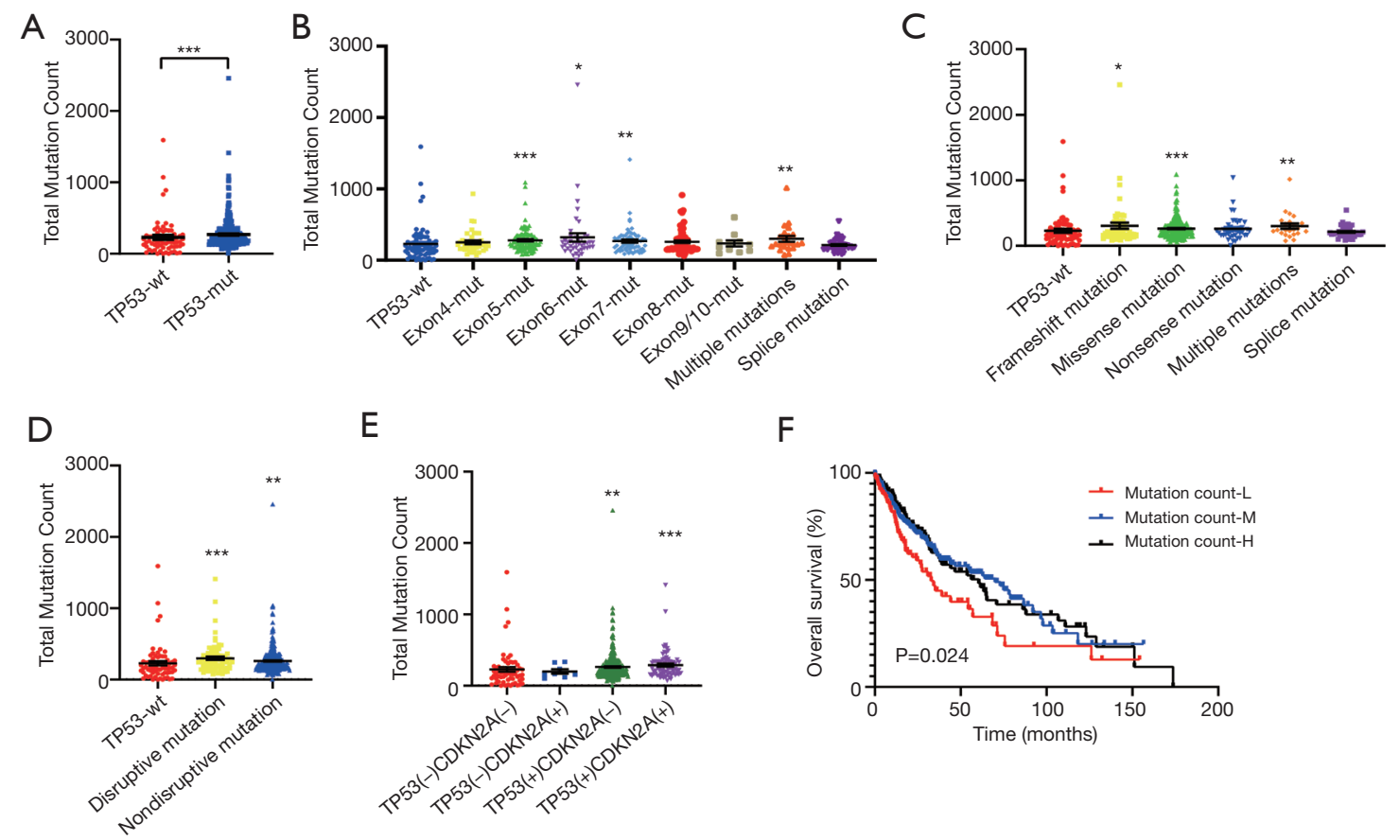

$E$

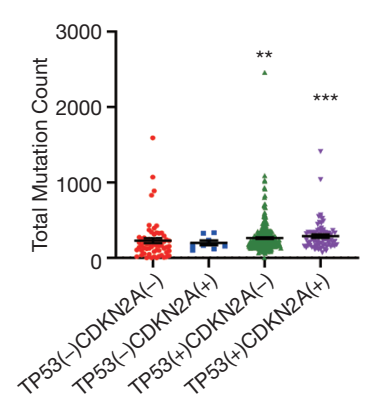

$\mathrm{F}$

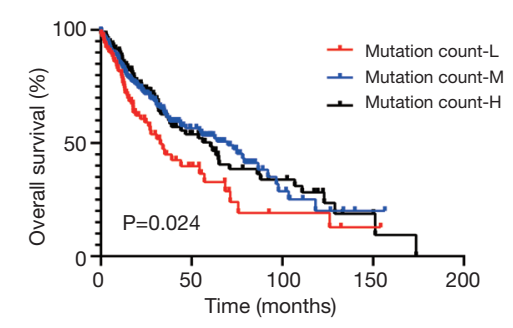

Figure 5 The correlation between mutation counts and tumor protein p53 (TP53) status and survival time. (A) Total mutation count in LUSC patients with wild-type and mutated TP53. (B) Total mutation count in lung squamous cell carcinoma (LUSC) patients with wildtype and mutated TP53 subdivided according to mutation site. (C) Total mutation count in LUSC patients with wild-type and mutated P53 subdivided according to mutation type. (D) Total mutation count in LUSC patients with wild-type and mutated P53 subdivided into disruptive or nondisruptive mutation types. (E) Total mutation count in LUSC patients with different cyclin dependent kinase inhibitor 2A (CDKN2A)/TP53 mutations. (F) Overall survival (OS) of early-stage LUSC patients subdivided into a low mutation count group (mutation count-L), a medium mutation count group (mutation count-M), and a high mutation count group (mutation count-H). For (A-E), each dot represents a patient $[$ mean \pm standard deviation $(\mathrm{SD})] ;{ }^{*}, \mathrm{P}<0.05 ;{ }^{* *}, \mathrm{P}<0.01 ;{ }^{* * *}, \mathrm{P}<0.001$.

mutation cohort had more mutations irrespective of CDKN2A mutation status. These results suggested that mutation count is associated with TP53 status, independent of CDKN2A status (Figure 5E). The number of mutations was divided into 3 cohorts, namely, the low mutation count cohort, which included patients with 1-150 mutations; the medium mutation count cohort, which included patients with 151-300 mutations; and the high mutation count cohort, which included patients with over 301 mutations. OS was significantly shorter in the low mutation count cohort compared to patients in the medium and high mutation count groups $(\mathrm{P}=0.024$; Figure $5 F)$.

\section{Discussion}

In LUSC, recurrent mutations of TP53, FGFR1, FGFR2, FGFR3, DDR2, and genes of the PI3K pathway have been detected, as have quantitative gene abnormalities of PTEN and CDKN2A $(1,6)$. This current study reviewed 16 patients with surgically resected LUSC and identified that TP53 and CDKN2A exhibited a higher frequency of somatic mutations than other cancer-related genes. These results were compared with those from the TCGA dataset, which is mainly composed of the Western population. Therefore, it is essential to further elucidate the association of TP53 status and CDKN2A status, as well as the prognostic value of these two genes together in earlystage, surgically resected LUSC patients in the Chinese population .

TP53 has been shown to be one of the most frequently mutated genes in lung cancers irrespective of histological type, with the vast majority of mutations clustering in exons 4 to 8 (11), which is consistent with our study. In addition, similar to the results of previous studies (3), 
missense mutations were the most common mutations observed in our early-stage LUSC cohort. In contrast to other studies (11), we demonstrated that TP53 mutations were not significantly associated with age or stage. As we only analyzed the influence of TP53 mutations in relation to early-stage, surgically resected LUSC rather than NSCLC, this may account for the difference observed between studies. Interestingly, CDKN2A mutation status was shown to be associated with TP53 mutation status, and in fact, CDKN2A mutations were present in $17 \%$ of early-stage LUSCs. However, there was no significant relationship between CDKN2A mutation status and tumor characteristics.

To date, data on the prognostic or predictive effect of TP53 in NSCLC have been limited and inconclusive. In a study cohort of 35 patients with NSCLC from a prospective phase II trial, TP53 mutation was predictive of resistance to induction therapy (cisplatin/etoposide plus radiation) (12). However, Schiller et al. failed to identify prognostic or predictive value in 197 patients with completely resected tumors enrolled in a randomized trial of postoperative radiotherapy plus chemotherapy (13). Negative results were also observed in JBRonchus (JBR), a randomized trial of patients with stage IB and II NSCLC assigned to treatment with cisplatin-based adjuvant chemotherapy (ACT) versus observation (OBS) (14). Another randomized trial of ACT versus OBS in patients with stage I to III NSCLC, the International Adjuvant Lung Cancer Trial (IALT), showed that TP53 mutation was neither prognostic nor predictive for OS after 8 years of follow-up. Ma et al. performed a pooled analysis of four randomized trials of ACT versus OBS and reported that TP53 mutation had no prognostic effect but was marginally predictive for survival from ACT (4).

This current investigation examined the prognostic value of TP53 in early-stage LUSC. Analysis of the TCGA data revealed a trend towards decreased OS with progressing tumor stage, regardless of TP53 status. Our study indicated that TP53mutation is a favorable prognostic factor in earlystage LUSC patients. This effect was only significant in stage III patients and not in stage I-II patients. OS was also significantly affected by the year of initial diagnosis, especially before 2010, and TP53 status. This discrepancy might be attributable to the development of ACT for use after surgery. TP53 mutations should be considered not only in terms of mutation status but also in terms of mutation site and mutation type. We found that the TP53 mutation site and mutation type were clinically meaningful. Similar to previous studies (15), patients with TP53 exon
4 or exon 6 mutations demonstrated poorer prognosis compared to patients with TP53 exon 5 , exon 7 , or exon 8 mutations. In addition, patients with multiple mutations demonstrated better prognosis than those with nonsense mutations. The former study divided TP53 mutation types into disruptive and nondisruptive, and found that nondisruptive mutations of TP53 are an independent prognostic factor of shorter survival time in EGFR-mutated NSCLC (16). However, our study showed that disruptive mutation of TP53 seemed to confer a longer survival time in early-stage LUSC, in agreement with Hou et al. (17).

CDKN2A alterations are frequent in all lung cancer expression subtypes (6). However, few reports have investigated the predictive or prognostic significance of CDKN2A in NSCLC. Our study indicated that CDKN2A mutations in early-stage LUSC are significantly associated with poor survival time. This was also the first study to analyze the association between TP53 status and CDKN2A status in early-stage, surgically resected LUSC patients. Patients with mutated TP53 and wild-type CDKN2A demonstrated a longer survival time compared with other early-stage LUSC patients. When CDKN2A status was divided into wild-type and mutated groups, survival curves of the mutated CDKN2A group showed that TP53 wild-type patients had a poorer prognosis. There were no significant differences between wild-type CDKN2A and TP53 status in terms of OS. The results suggested that CDKN2A mutation is a vital indicator for prognostic assessment according to TP53 status.

This investigation demonstrated that patients with TP53 mutations have longer OS and DFS among earlystage LUSC patients. Patients with TP53 mutation had more total mutations than those with wild-type TP53. Specifically, patients with different TP53 mutation sites and mutation types harbored different mutation counts and had higher mutation counts than those with wild-type TP53. Interestingly, patients with higher mutation counts had a longer survival time, which was consistent with the results demonstrating that patients with TP53 mutations had a longer survival time. Previous reports have suggested that the measurement of mutation counts is representative of tumor mutation burden (TMB) $(18,19)$. Tumors with high TMB are thought to express more cancer-specific antigens (neoantigens) that can be recognized by the immune system (20). In the present study, data from the TCGA database included information on patients with early-stage, surgically resected LUSC from 1992 to 2013 who had good performance status and an active immune 
system. Moreover, patients with TP53 mutations diagnosed before 2010 who accepted limited therapy after surgery had a longer survival time than P53-wild-type patients. This discrepancy might be caused by differences in stage III patients and stage I-II patients. As resectable stage III LUSC has more circulating tumor cells, and patients with TP53 mutations may harbor higher mutation counts and express more neoantigens which can be recognized by the immune system compared to patients without TP53 mutations.

There were several limitations to this investigation including the small sample size of the cohort and inadequate information from the cBioPortal database. Future work should verified these results using other cohorts, such as data from the TCGA cohort. Further investigations regarding TP53 and CDKN2A mutations, and the prognosis of LUSC patients are required to fully evaluate the role of TP53/ CDKN2A status as a prognostic and predictive variable in patients with LUSC. Though many biochemical aspects of p53 and CDKN2A regulation and activity were elucidated and it have demonstrated their inhibition of tumorigenesis $(21,22), \mathrm{p} 53$ and CDKN2A mutants differ considerably in form and function need furthermore investigation. We hope that different treatment strategies were adopted according to TP53 and CDKN2A status.

\section{Acknowledgments}

Funding: This work was supported by funding from a specific research fund for the public service sector, the National Natural Science Foundation of China (Grant Number 82002497), and the Science and Technology Program of Fujian Province (Grant Number 2020J05072).

\section{Footnote}

Reporting Checklist: The authors have completed the REMARK reporting checklist. Available at https://dx.doi. org/10.21037/atm-21-3709

Data Sharing Statement: Available at https://dx.doi. org/10.21037/atm-21-3709

Conflicts of Interest: All authors have completed the ICMJE uniform disclosure form (available at https://dx.doi. org/10.21037/atm-21-3709). The authors report funding support from the National Natural Science Foundation of China (Grant Number 82002497), and the Science and
Technology Program of Fujian Province (Grant Number 2020J05072) for the article processing charges. The authors have no other conflicts of interest to declare.

Ethical Statement: The authors are accountable for all aspects of the work in ensuring that questions related to the accuracy or integrity of any part of the work are appropriately investigated and resolved. All procedures performed in this study involving human participants were in accordance with the Declaration of Helsinki (as revised in 2013). The study was approved by regional ethics board of Fujian Cancer Hospital (No.: SQ2020-055-01) and informed consent was taken from all the patients.

Open Access Statement: This is an Open Access article distributed in accordance with the Creative Commons Attribution-NonCommercial-NoDerivs 4.0 International License (CC BY-NC-ND 4.0), which permits the noncommercial replication and distribution of the article with the strict proviso that no changes or edits are made and the original work is properly cited (including links to both the formal publication through the relevant DOI and the license). See: https://creativecommons.org/licenses/by-nc-nd/4.0/.

\section{References}

1. Choi M, Kadara H, Zhang J, et al. Mutation profiles in early-stage lung squamous cell carcinoma with clinical follow-up and correlation with markers of immune function. Ann Oncol 2017;28:83-9.

2. Cancer Genome Atlas Research Network. Comprehensive genomic characterization of squamous cell lung cancers. Nature 2012;489:519-25.

3. Bykov VJN, Eriksson SE, Bianchi J, et al. Targeting mutant p53 for efficient cancer therapy. Nat Rev Cancer 2018;18:89-102.

4. Ma X, Le Teuff G, Lacas B, et al. Prognostic and Predictive Effect of TP53 Mutations in Patients with Non-Small Cell Lung Cancer from Adjuvant CisplatinBased Therapy Randomized Trials: A LACE-Bio Pooled Analysis. J Thorac Oncol 2016;11:850-61.

5. Helgadottir H, Höiom V, Tuominen R, et al. Germline CDKN2A Mutation Status and Survival in Familial Melanoma Cases. J Natl Cancer Inst 2016. doi: 10.1093/ jnci/djw135.

6. Testa U, Castelli G, Pelosi E. Lung Cancers: Molecular Characterization, Clonal Heterogeneity and Evolution, and Cancer Stem Cells. Cancers (Basel) 2018;10:248. 
7. Wang J, Ding S, Duan Z, et al. Role of p14ARFHDM2-p53 axis in SOX6-mediated tumor suppression. Oncogene 2016;35:1692-702.

8. Heo SH, Kwak J, Jang KL. All-trans retinoic acid induces p53-depenent apoptosis in human hepatocytes by activating p14 expression via promoter hypomethylation. Cancer Lett 2015;362:139-48.

9. Poeta ML, Manola J, Goldwasser MA, et al. TP53 mutations and survival in squamous-cell carcinoma of the head and neck. N Engl J Med 2007;357:2552-61.

10. Soussi T, Wiman KG. TP53: an oncogene in disguise. Cell Death Differ 2015;22:1239-49.

11. Ma X, Rousseau V, Sun H, et al. Significance of TP53 mutations as predictive markers of adjuvant cisplatin-based chemotherapy in completely resected non-small-cell lung cancer. Mol Oncol 2014;8:555-64.

12. Kandioler D, Stamatis G, Eberhardt W, et al. Growing clinical evidence for the interaction of the p53 genotype and response to induction chemotherapy in advanced non-small cell lung cancer. J Thorac Cardiovasc Surg 2008;135:1036-41.

13. Schiller JH, Adak S, Feins RH, et al. Lack of prognostic significance of $\mathrm{p} 53$ and K-ras mutations in primary resected non-small-cell lung cancer on E4592: a Laboratory Ancillary Study on an Eastern Cooperative Oncology Group Prospective Randomized Trial of Postoperative Adjuvant Therapy. J Clin Oncol 2001;19:448-57.

14. Tsao MS, Aviel-Ronen S, Ding K, et al. Prognostic and predictive importance of p53 and RAS for adjuvant chemotherapy in non small-cell lung cancer. J Clin Oncol 2007;25:5240-7.

Cite this article as: Wang $\mathrm{P}$, Wang $\mathrm{F}$, He H, Chen Y, Lin H, Chen P, Chen X, Liu S. TP53 and CDKN2A mutations in patients with early-stage lung squamous cell carcinoma: an analysis of the correlations and prognostic outcomes. Ann Transl Med 2021;9(16):1330. doi: 10.21037/atm-21-3709
15. Jiao XD, Qin BD, You P, et al. The prognostic value of TP53 and its correlation with EGFR mutation in advanced non-small cell lung cancer, an analysis based on cBioPortal data base. Lung Cancer 2018;123:70-5.

16. Canale M, Petracci E, Delmonte A, et al. Impact of TP53 Mutations on Outcome in EGFR-Mutated Patients Treated with First-Line Tyrosine Kinase Inhibitors. Clin Cancer Res 2017;23:2195-202.

17. Hou H, Qin K, Liang Y, et al. Concurrent TP53 mutations predict poor outcomes of EGFR-TKI treatments in Chinese patients with advanced NSCLC. Cancer Manag Res 2019;11:5665-75.

18. Oh JH, Jang SJ, Kim J, et al. Spontaneous mutations in the single TTN gene represent high tumor mutation burden. NPJ Genom Med 2020;5:33.

19. Endris V, Buchhalter I, Allgäuer M, et al. Measurement of tumor mutational burden (TMB) in routine molecular diagnostics: in silico and real-life analysis of three larger gene panels. Int J Cancer 2019;144:2303-12.

20. Zhang H, Deng YM, Chen ZC, et al. Clinical significance of tumor mutation burden and DNA damage repair in advanced stage non-small cell lung cancer patients. Eur Rev Med Pharmacol Sci 2020;24:7664-72.

21. Hernández Borrero LJ, El-Deiry WS. Tumor suppressor p53: Biology, signaling pathways, and therapeutic targeting. Biochim Biophys Acta Rev Cancer 2021;1876:188556.

22. Adib E, Nassar AH, Akl EW, et al. CDKN2A Alterations and Response to Immunotherapy in Solid Tumors. Clin Cancer Res 2021;27:4025-35.

(English Language Editor: J. Teoh) 

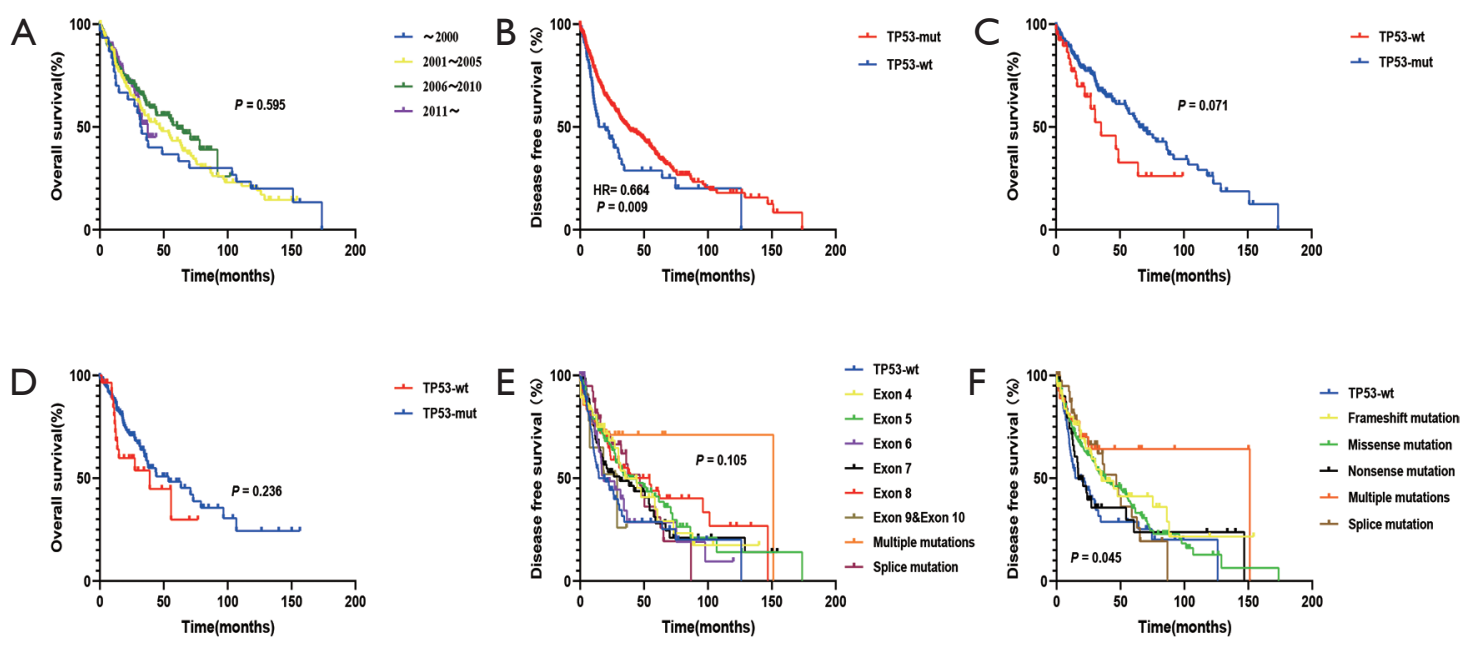

Figure S1 Survival curves of patients carrying tumor protein p53 (TP53) mutations. (A) Overall survival (OS) of patients with different year of initial diagnosis according to tumor staging. (B) Disease free survival (DFS) in TP53 wild-type and mutated patients. (C) OS in stage I patients carrying wild-type or mutated TP53. (D) OS in stage II patients carrying wild-type or mutated TP53. (E) DFS in patients with wild-type and mutated TP53 subdivided according to mutation site. (F) DFS in patients with wild-type and mutated TP53 subdivided according to mutation type.

A

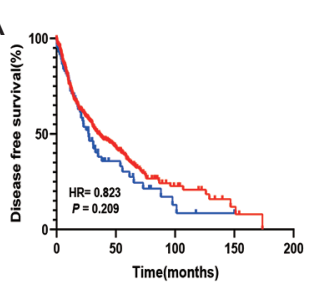

D

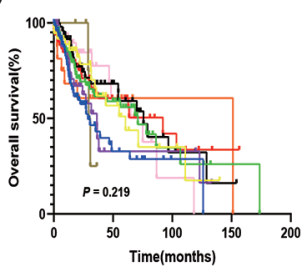

G

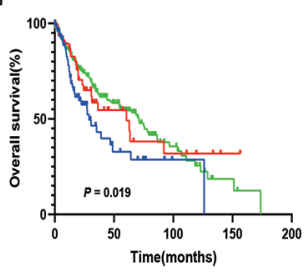

B
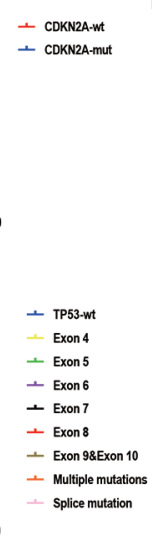

$\mathrm{E}$

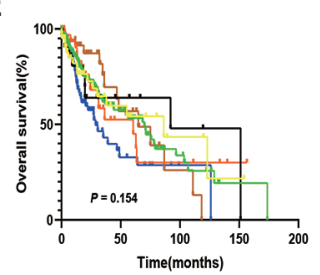

C
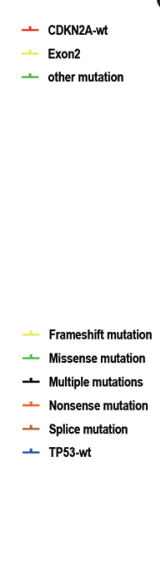

$\mathrm{F}$
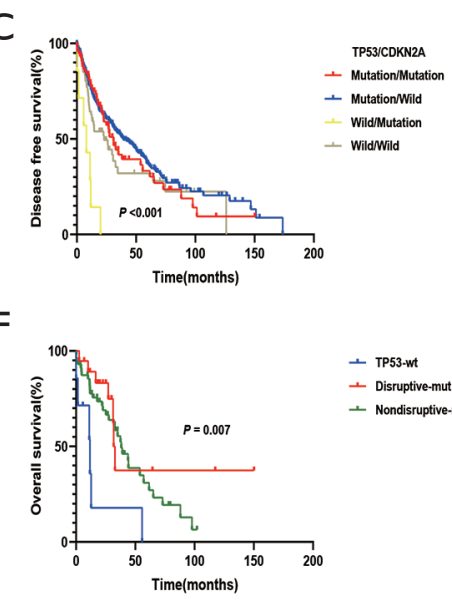

- TP53-wt

$\perp$ Disruptive-mut
$\perp$ Nondisuptive-mut

Figure S2 Survival curves of patients carrying cyclin dependent kinase inhibitor 2A (CDKN2A) and tumor protein p53 (TP53) mutations. (A) Disease free survival (DFS) in patients with wild-type and mutated CDKN2A. (B) DFS in patients with wild-type and mutated CDKN2A subdivided according to mutation site. (C) DFS of patients in different CDKN2A/TP53 mutation groups. (D) Overall survival (OS) of different TP53 mutation sites in CDKN2A wild-type patients. (E) OS of different CDKN2A mutation types in CDKN2A wild-type patients. (F) OS of patients with wild-type and mutated TP53 subdivided into disruptive or nondisruptive mutations in CDKN2A-mutated patients. (G) OS of CDKN2A wild-type patients with wild-type and mutated TP53 subdivided into disruptive or nondisruptive mutations. 

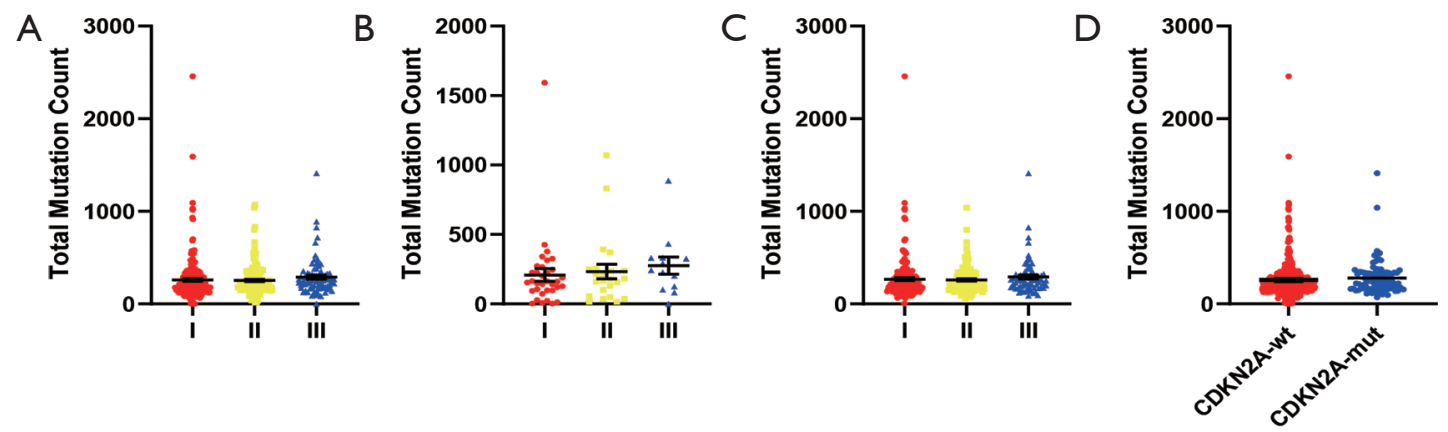

Figure S3 The mutation counts of LUSC patients. (A) Total mutation count of patients with lung squamous cell carcinoma (LUSC) at different tumor stages. (B) Total mutation count of TP53 wild-type patients with LUSC at different tumor stages. (C) Total mutation count of TP53-mutated patients with LUSC at different tumor stages. (D) Total mutation count of LUSC patients with wild-type and mutated CDKN2A. For (A-D), each dot represents a patient [mean \pm standard deviation (SD)].

Table S1 1,021 gene panel

\begin{tabular}{|c|c|c|c|c|c|c|c|c|}
\hline \multicolumn{9}{|c|}{ whole exons } \\
\hline ARAF & ARID1A & ARID1B & ARID2 & ASXL1 & ATM & ATR & ATRX & AURKA \\
\hline AURKB & AXIN1 & AXIN2 & $A X L$ & B2M & BAP1 & BARD1 & BCL2 & BCL2L1 \\
\hline BCOR & BLM & BMPR1A & BRAF & BRCA1 & BRCA2 & BRD4 & BRIP1 & BTK \\
\hline CDC73 & $\mathrm{CDH} 1$ & CDK12 & CDK4 & CDK6 & CDK8 & CDKN1A & CDKN1B & CDKN2A \\
\hline CDKN2B & CDKN2C & CEBPA & CHEK1 & CHEK2 & $\mathrm{CIC}$ & CREBBP & CRKL & CSF1R \\
\hline CTCF & CTNNA1 & CTNNB1 & CUL3 & CYLD & DAXX & DDR1 & DDR2 & DICER1 \\
\hline DNMT3A & EGFR & ELAC2 & EME2 & EP300 & EPAS1 & EPCAM & EPHA2 & ЕРНАЗ \\
\hline FANCD2 & FANCG & FANCM & FAS & FAT1 & FAT2 & FBXW7 & FCGR2A & FCGR3A \\
\hline FGFR1 & FGFR2 & FGFR3 & FGFR4 & $\mathrm{FH}$ & FLCN & FLT1 & FLT3 & FLT4 \\
\hline FOXA1 & FOXL2 & FOXP1 & FUBP1 & GAB2 & GALNT12 & GATA3 & GNA11 & GNAQ \\
\hline GNAS & GRIN2A & HDAC1 & HDAC4 & HGF & HNF1A & HOXB13 & HRAS & HSP90AA1 \\
\hline IDH1 & IDH2 & IFNG & IFNGR1 & IGF1R & IL7R & INPP4B & IRF2 & IRS2 \\
\hline JAK1 & JAK2 & JAK3 & KDM5A & KDM5C & KDM6A & KDR & KEAP1 & $\mathrm{KIT}$ \\
\hline KRAS & LRP1B & MAP2K1 & MAP2K2 & MAP2K4 & MAP3K1 & MAPK1 & MAX & MCL1 \\
\hline MDM2 & MDM4 & MED12 & MEN1 & MET & MITF & MLH1 & MLH3 & MLL \\
\hline
\end{tabular}

Table S1 (continued) 
Table S1 (continued)

\begin{tabular}{|c|c|c|c|c|c|c|c|c|}
\hline \multicolumn{9}{|l|}{ whole exons } \\
\hline PDK1 & PHF6 & PIK3CA & РІКЗСВ & PIK3CG & PIK3R1 & PIK3R2 & PMS1 & PMS2 \\
\hline POLD1 & POLE & POT1 & PPM1D & PRKAR1A & PTCH1 & РTCH2 & PTEN & PTPN11 \\
\hline RAD50 & RAD51 & RAD51B & RAD51C & RAD51D & RAF1 & RARA & RB1 & RBM10 \\
\hline RUNX1 & SDHA & SDHAF2 & SDHB & SDHC & SDHD & SERPINB3 & SERPINB4 & SETD2 \\
\hline SLX4 & SMAD2 & SMAD4 & SMARCA4 & SMARCB1 & SMARCE1 & SMO & sox2 & sox9 \\
\hline SRC & STAG2 & STAT3 & STK11 & SUFU & SYK & TBX3 & TCF7L2 & TET2 \\
\hline TGFBR2 & TMEM127 & TMPRSS2 & TNFAIP3 & TOP1 & TOP2A & TP53 & TP73 & TSC1 \\
\hline ALK & BCL2L11 & BRAF & BRCA1 & BRD4 & CD74 & EGFR & EML4 & ERG \\
\hline ETV6 & EZR & FGFR1 & FGFR2 & FGFR3 & KIF5B & KIT & MAML2 & MET \\
\hline MSH2 & MYC & MYCL1 & NCOA4 & NOTCH2 & NTRK1 & NTRK2 & NTRK3 & PDGFRA \\
\hline PMS2 & PPARG & RAF1 & RET & ROS1 & RSPO2 & SLC34A2 & TERT & TFE3 \\
\hline $\begin{array}{c}\text { TMPRSS2 } \\
\text { partial exons }\end{array}$ & TPM3 & & & & & & & \\
\hline ABCA13 & ABCB1 & $\mathrm{ABCC} 1$ & $\mathrm{ABCC} 11$ & $\mathrm{ABCC} 2$ & ABCG2 & ACACA & ACIN1 & АСТВ \\
\hline ACTG1 & ACTG2 & ACVR2A & ACVRL1 & ADAM29 & ADAMTS5 & ADCY1 & AFF1 & AFF2 \\
\hline BIRC2 & BIRC3 & BMPR2 & BNC2 & BPTF & BRD2 & BRD3 & BRSK1 & BRWD1 \\
\hline BTLA & BUB1 & C15orf23 & C15orf55 & C1QA & C1S & C3orf70 & C7orf53 & C8orf34 \\
\hline CACNA1D & CACNA1E & CADM2 & CAMTA1 & CAPN7 & CARD11 & CASP1 & CASQ2 & CBLB \\
\hline CBR1 & CBR3 & CCDC168 & CCNA1 & CCNB3 & ССТ3 & ССТ5 & ССт6В & CD22 \\
\hline CD33 & CD5L & CDA & $\mathrm{CDH} 11$ & $\mathrm{CDH} 18$ & $\mathrm{CDH} 23$ & CDK13 & CHD1 & CHD1L \\
\hline CHD3 & CHD4 & CHD6 & CHD8 & CHD9 & CHFR & CHI3L1 & CHN1 & CIITA \\
\hline CKS1B & CLCC1 & CLDN18 & CLP1 & CLSPN & CLTC & CNOT3 & CNOT4 & CNTN1 \\
\hline CNTN5 & CNTNAP1 & CNTNAP5 & COL1A1 & COL2A1 & COL5A1 & COL5A2 & COL5A3 & COPS2 \\
\hline CPS1 & CREB3L1 & CRIPAK & CRLF2 & CRNKL1 & CRTC1 & CRYBG3 & CSF1 & CSF3R \\
\hline CSMD1 & CSMD3 & CSNK1A1 & CSNK1G3 & CSNK2A1 & CTLA4 & CTNNA2 & CTNND1 & CUX1 \\
\hline CYBA & CYP19A1 & CYP1B1 & CYP1A1 & CYP2A13 & CYP2C19 & CYP2C8 & CYP2D6 & CYP3A4 \\
\hline
\end{tabular}

Table S1 (continued) 
Table S1 (continued)

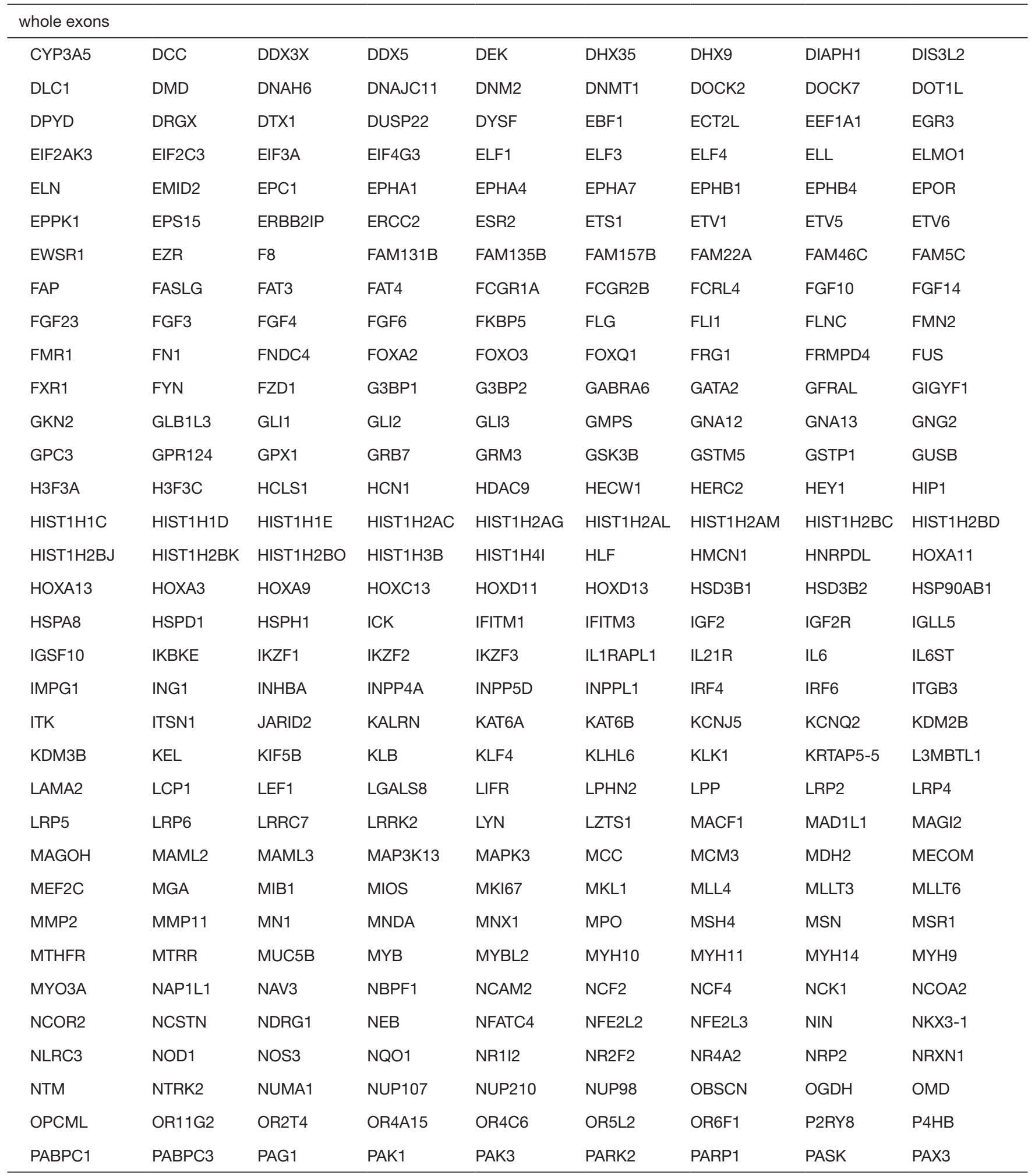

Table S1 (continued) 
Table S1 (continued)

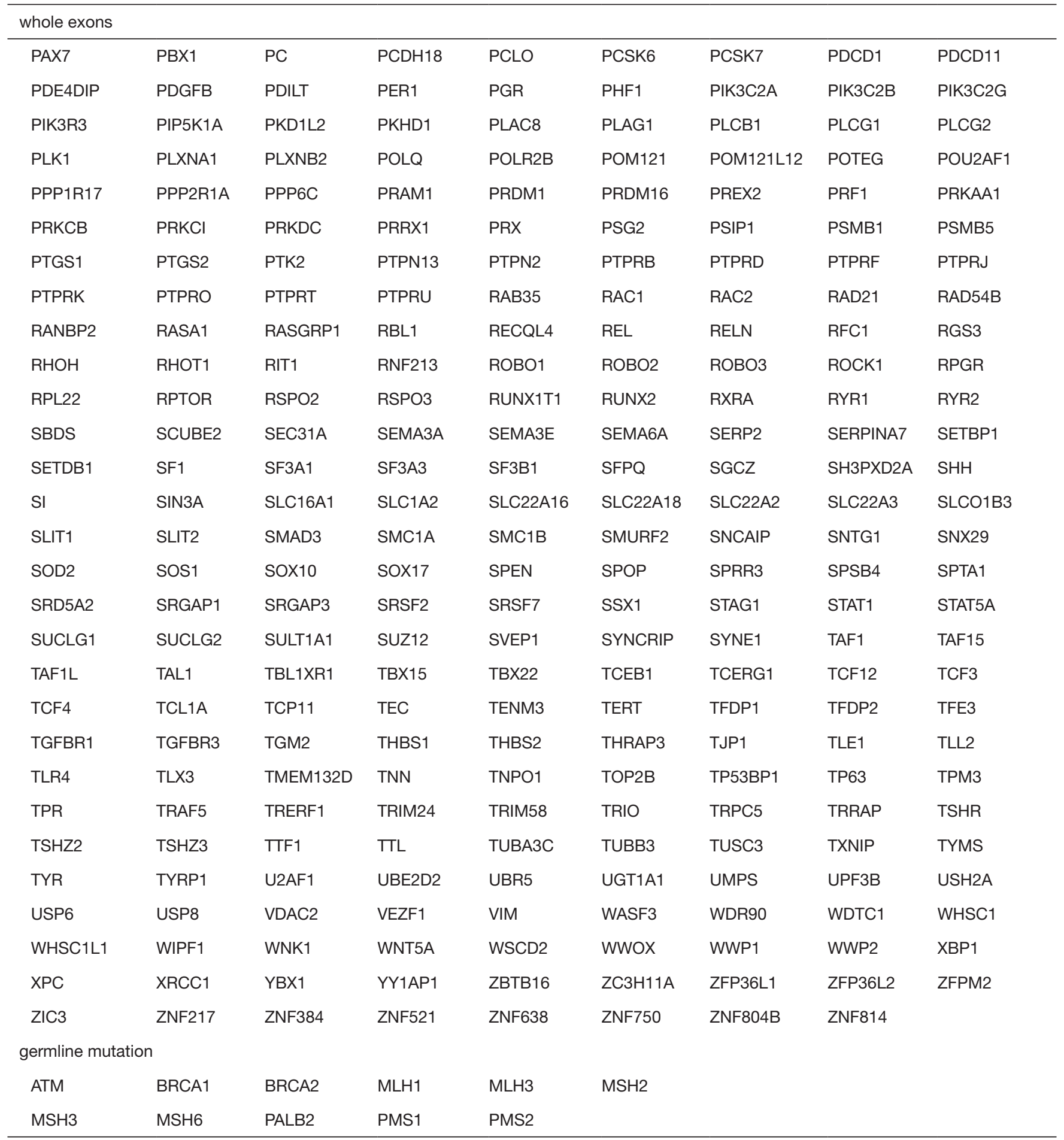


Table S2 Patient and tumor characteristics according to TP53 status

\begin{tabular}{|c|c|c|c|c|}
\hline Variable & $\mathrm{N}$ & \multicolumn{3}{|c|}{ TP53 status } \\
\hline \multicolumn{5}{|l|}{ Age (years) } \\
\hline$<60$ & 89 & 74 & 15 & 0.452 \\
\hline $60-70$ & 204 & 174 & 30 & \\
\hline Unknown & 9 & 8 & 1 & \\
\hline \multicolumn{5}{|l|}{ Sex } \\
\hline Male & 363 & 307 & 56 & 0.152 \\
\hline Female & 129 & 102 & 27 & \\
\hline \multicolumn{5}{|c|}{ Lymph node status } \\
\hline $\mathrm{pN} 1$ & 130 & 105 & 25 & \\
\hline $\mathrm{pN} 2 / \mathrm{N} 3$ & 46 & 39 & 7 & \\
\hline \multicolumn{5}{|l|}{ Tumor } \\
\hline $\mathrm{T} 1$ & 110 & 87 & 23 & 0.355 \\
\hline $\mathrm{T} 2$ & 288 & 245 & 43 & \\
\hline T3 & 70 & 59 & 11 & \\
\hline $\mathrm{T} 4$ & 24 & 18 & 6 & \\
\hline \multicolumn{5}{|l|}{ Tumor stage } \\
\hline \multicolumn{5}{|c|}{ Primary Tumor Site } \\
\hline L-Upper & 131 & 105 & 26 & 0.308 \\
\hline L-Lower & 77 & 62 & 15 & \\
\hline R-Upper & 128 & 107 & 21 & \\
\hline R-Middle & 17 & 12 & 5 & \\
\hline R-Lower & 108 & 96 & 12 & \\
\hline Bronchial & 10 & 9 & 1 & \\
\hline Unknown & 21 & 18 & 3 & \\
\hline
\end{tabular}

Table S2 (continued) 
Table S2 (continued)

\begin{tabular}{|c|c|c|c|c|}
\hline \multirow{2}{*}{ Variable } & \multirow{2}{*}{$\mathrm{N}$} & \multicolumn{3}{|c|}{ TP53 status } \\
\hline & & Mutation $(n=409)$ & Wild $(n=83)$ & $P$ value \\
\hline \multicolumn{5}{|c|}{ Year Initial Diagnosis } \\
\hline-2000 & 30 & 24 & 6 & 0.583 \\
\hline $2001-2005$ & 96 & 85 & 11 & \\
\hline $2006-2010$ & 182 & 151 & 31 & \\
\hline $2011-$ & 167 & 135 & 32 & \\
\hline Unknown & 17 & 14 & 3 & \\
\hline \multicolumn{5}{|c|}{ Surgical Margin Resection Status } \\
\hline Ro & 390 & 326 & 64 & 0.862 \\
\hline $\mathrm{R} 1+\mathrm{R} 2$ & 17 & 14 & 3 & \\
\hline Unknown & 85 & 69 & 16 & \\
\hline \multicolumn{5}{|c|}{ CDKN2A Mutation status } \\
\hline Wild type & 406 & 331 & 75 & 0.040 \\
\hline Mutated type & 86 & 78 & 8 & \\
\hline
\end{tabular}


Table S3 Patient and tumor characteristics according to CDKN2A status

\begin{tabular}{|c|c|c|c|c|}
\hline \multirow{2}{*}{ Variable } & \multirow{2}{*}{$N$} & \multicolumn{3}{|c|}{ CDKN2A status } \\
\hline & & Mutation $(n=86)$ & Wild $(n=406)$ & $P$ value \\
\hline \multicolumn{5}{|l|}{ Age (years) } \\
\hline$<60$ & 89 & 20 & 69 & 0.553 \\
\hline $60-70$ & 210 & 34 & 176 & \\
\hline$>70$ & 184 & 30 & 154 & \\
\hline Unknown & 9 & 2 & 7 & \\
\hline \multicolumn{5}{|l|}{ Sex } \\
\hline Male & 363 & 65 & 298 & 0.418 \\
\hline Female & 129 & 21 & 108 & \\
\hline \multicolumn{5}{|c|}{ Lymph node status } \\
\hline pNO & 316 & 53 & 263 & 0.225 \\
\hline $\mathrm{pN} 1$ & 130 & 28 & 102 & \\
\hline $\mathrm{pN} 2 / \mathrm{N} 3$ & 46 & 5 & 41 & \\
\hline \multicolumn{5}{|l|}{ Tumor } \\
\hline T1 & 110 & 17 & 93 & 0.844 \\
\hline T2 & 288 & 53 & 235 & \\
\hline T3 & 70 & 11 & 59 & \\
\hline $\mathrm{T} 4$ & 24 & 5 & 19 & \\
\hline \multicolumn{5}{|c|}{ Tumor stage } \\
\hline I & 239 & 38 & 201 & 0.344 \\
\hline II & 160 & 34 & 126 & \\
\hline III & 86 & 12 & 74 & \\
\hline IV & 7 & 2 & 5 & \\
\hline \multicolumn{5}{|c|}{ Primary Tumor Site } \\
\hline L-Upper & 131 & 20 & 111 & 0.332 \\
\hline L-Lower & 77 & 13 & 64 & \\
\hline R-Upper & 128 & 22 & 106 & \\
\hline R-Middle & 17 & 1 & 16 & \\
\hline R-Lower & 108 & 20 & 88 & \\
\hline Bronchial & 10 & 3 & 7 & \\
\hline Unknown & 21 & 7 & 14 & \\
\hline
\end{tabular}

